\title{
Stationary solutions for a model of amorphous thin-film growth
}

October 25, 2018

\author{
Dirk Blömker ${ }^{1}$ and Martin Hairer ${ }^{2}$ \\ ${ }^{1}$ Institut für Mathematik, RWTH Aachen, Germany \\ Email: bloemker@instmath.rwth-aachen.de \\ ${ }^{2}$ Département de Physique Théorique, University of Geneva, Switzerland \\ Email: Martin.Hairer@physics.unige.ch
}

\begin{abstract}
We consider a class of stochastic partial differential equations arising as a model for amorphous thin film growth. Using a spectral Galerkin method, we verify the existence of stationary mild solutions, although the specific nature of the nonlinearity prevents us from showing the uniqueness of the solutions as well as their boundedness (in time).
\end{abstract}

\section{Introduction}

This paper shows the existence of a stationary solution for a stochastic partial differential equation (SPDE), where the solutions may not form a Markov semigroup due to the lack of uniqueness. We consider the family of equations

$$
\partial_{t} u=-\partial_{x}^{4} u+\nu \partial_{x}^{2} u-\partial_{x}^{2}\left(\partial_{x} u\right)^{2}+\xi(x, t), \quad \nu \in \mathbf{R},
$$

for a real-valued scalar $u(t, x)$ with $t>0$ and $x \in[0, L]$, subject to suitable boundary conditions (e.g. periodic or Neumann type). The symbol $\xi$ denotes a noise process which should be thought of as the generalized derivative of some Wiener process to be specified later on.

Equations of the type (1.1) arise in the growth of thin films (see e.g. $\mathrm{RML}^{+} 00$, SP94, BS95]). The function $u(t, \cdot)$ describes the graph of a surface at time $t>0$. Usually these equations are equipped with a lot of physical parameters, which we set to 1 for simplicity. In some models an additional additive nonlinear term $\left(\partial_{x} u\right)^{2}$ appears. We can treat that case too, but the analysis is more involved without contributing much to the general understanding of the situation, so we do not present it.

It is easy to verify that there exists a value $\nu_{c}<0$ such that if $\nu<\nu_{c}$, the equations under consideration present a linear instability. We will therefore distinguish in the sequel the stable case $\left(\nu>\nu_{c}\right)$ from the unstable case $\left(\nu \leq \nu_{c}\right)$. This instability is responsible for the formation of hills, which is frequently seen in experiments (see 
e.g. MMS99] and the references therein). On the other hand we have a quadratic nonlinearity that compensates this instability. Unfortunately, this nonlinearity makes it difficult to derive uniform bounds on the solution. Moreover, it is an open problem how to establish bounds in case of a two-dimensional square, which is obviously a more realistic model than the one-dimensional case we treat in this paper. This scenario is similar to the Kuramoto-Sivashinsky equation, where there are no results for truly twodimensional domains.

One very helpful tool in the analysis is the conservation of mass:

Remark 1.1 The quantity $M(u):=\int_{0}^{L} u(x) d x$ decouples from the rest of the equation. Therefore, we can assume without loss of generality that $M(u(t)) \equiv 0$ for a solution of (1.1). The various Sobolev spaces appearing in the sequel should be thought of as the orthogonal complement to the constant function 1 of the usual spaces.

The local existence of unique solutions to equations of the type (1.1) is standard for sufficiently smooth initial conditions. But the existence of global solutions is much more complicated, and was shown in [BG02] or [BGR02] using a spectral Galerkin method. Nevertheless the question of uniqueness of global solutions is still open, even in the deterministic case, as it is out of reach to show enough regularity. Therefore the equation does not necessarily generate a Markov semigroup, and the standard theory for invariant measures (cf. [DPZ96) does not apply.

We show in this paper that there exists nevertheless an "invariant measure" for 1.1. To be more precise, we construct a stationary solution $\{u(t), t \in \mathbf{R}\}$ such that the distribution $\mathcal{P}^{t}:=\mathcal{L}(u(t))$ of $u(t)$ is constant in time.

Our concept of solutions is a martingale solution of the corresponding mild formulation. Hence, we allow a change of the underlying probability space and consider solutions not of the SPDE, but of the corresponding variation of constants formula. Since we use spectral Galerkin methods, our approach is similar to previous results (see [FG95] or [CG94]) for the stochastic Navier-Stokes equation. One of the major differences is that we are not able to use the theory of Markov semigroups. Moreover, we were not able to get any uniform bound (in $t$ ) on the distribution of solutions when the driving force $\xi$ is a space-time white noise. We are able to establish such a bound only for stationary solutions. Therefore, we will construct the stationary process $u$ as a limit of the unique invariant stationary solutions to the Galerkin approximations.

The paper is organized as follows. In Section 2 , we introduce the spectral Galerkin approximation and present our main result. The next Section 3 presents compactness results and the proof of the main result. In Sections 4 and 5, we will give a-priori estimates for the solutions. The final Section 6 contains technical results.

\section{Acknowledgements}

D. B. wishes to thank Jean-Pierre Eckmanns mathematical physics group in Geneva for their warm hospitality. M. H. thanks Jean-Pierre Eckmann, Guillaume van Baalen, and Emmanuel Zabey for several discussions. The latter authors work was partially supported by the Fonds National Suisse.

\footnotetext{
${ }^{1}$ The symbol $\mathcal{L}(X)$ will always denote the law of the random variable $X$.
} 


\section{Notation and formulation of the main result}

Define the space $\mathrm{L}^{2}:=\left\{f \in \mathrm{L}^{2}([0, L]): \int_{0}^{L} f(x) d x=0\right\}$ with standard $\mathrm{L}^{2}$-norm $\|\cdot\|$. We define $A$ as the linear self-adjoint operator in $\mathrm{L}^{2}$ formally given by

$$
A:=-\partial_{x}^{4}+\nu \partial_{x}^{2},
$$

where the domain of definition $D(A)$ consists of all functions $f \in H^{4}([0, L])$ satisfying $M(f)=0$ and boundary conditions given by the equation. We will write $H^{4}=D(A)$ for short. Moreover, it is well-known that $A$ generates an analytic semigroup $\left\{e^{t A}\right\}_{t \geq 0}$, and we use the fractional powers of $A$ to define the standard fractional Sobolev spaces $H^{s}$ for $s \geq 0$ with dual spaces $H^{-s}$.

In the sequel we will need spaces of functions on the whole real line with values in Sobolev spaces. We recall the definition of the space $\mathcal{C}\left(\mathbf{R}, H^{s}\right)$, which is given as the set of all functions such that for any $a<b$, the restriction to $[a, b]$ is in $\mathcal{C}\left([a, b], H^{s}\right)$. We say that $f_{n}$ converges to $f$ in $\mathcal{C}\left(\mathbf{R}, H^{s}\right)$ if and only if $f_{n} \rightarrow f$ in any $\mathcal{C}\left([a, b], H^{s}\right)$ with $a<b$ equipped with the standard maximum norm. We define the spaces $\mathrm{L}_{\text {loc }}^{2}\left(\mathbf{R}, H^{s}\right)$ in an analogous way.

We write $\left\{e_{k}\right\}_{k \in \mathbf{N}}$ for a complete orthonormal set in $\mathrm{L}^{2}$ of eigenvectors of $A$ and denote by $\Pi_{N}$ the orthogonal projector onto the subspace of $\mathrm{L}^{2}$ spanned by $e_{1}, \ldots, e_{N}$. Then the $N$ th spectral Galerkin approximations $u_{N}$ of 1.1 is given by the solution of

$$
\partial_{t} u_{N}=A u_{N}+\Pi_{N} \partial_{x}^{2}\left(\partial_{x} u_{N}\right)^{2}+\Pi_{N} \dot{W},
$$

where $A$ can be interpreted either as an $N \times N$-matrix or as a differential operator acting on the range of $\Pi_{N}$. When considering (2.1), we will always take initial conditions in the range of $\Pi_{N}$. In this equation, $\dot{W}$ is the generalized time-derivative of a two-sided cylindrical Wiener process $W$ on $\mathrm{L}^{2}$ with covariance operator $Q$. (See [DPZ92] for the definition of a cylindrical Wiener process.) We make the following assumption on $Q$ :

Assumption 2.1 There exist positive numbers $\alpha_{k}$ and a constant $C$ such that

$$
Q e_{k}=\alpha_{k} e_{k} \quad \text { and } \quad\left|\alpha_{k}\right| \leq C,
$$

for all $k>0$.

Notice that this assumption covers the case of space-time white noise $\left(\alpha_{k}=1\right)$. The assumption that $Q$ and $A$ have the same eigenvectors implies that we restrict ourselves to translationally invariant noise, which is also called homogeneous in the physics literature. This assumption is crucial to verify technical results like Lemma 6.1.

Since (2.1) is actually a stochastic differential equation in $\mathbf{R}^{N}$ with locally Lipschitz coefficients, it is well-known (see e.g. [Has80] or [Arn74]) that it possesses (locally) a strong solution. Standard arguments allow to show the following proposition, the proof of which will be given in Section $\theta$ below.

Proposition 2.2 For every $N \geq 1$ and any initial condition in $\Pi_{N} \mathrm{~L}^{2}$, Equation (2.1) possesses a unique global strong solution. Furthermore, the law of this solution converges in variation norm towards a unique invariant measure $\mathcal{Q}_{N}$ which has bounded moments of second order. 
Consider processes $\left\{u_{N}(t)\right\}_{t \in \mathbf{R}}$ given as stationary solutions of the $N$ th spectral Galerkin approximation corresponding to the invariant measure $\mathcal{Q}_{N}$. Hence, $u_{N}$ satisfies the following stochastic ODE.

$$
\partial_{t} u_{N}=A u_{N}+\Pi_{N} \partial_{x}^{2}\left(\partial_{x} u_{N}\right)^{2}+\dot{W}_{N},
$$

where $W_{N}$ is given by $W_{N}(t)=\sum_{k=1}^{N} \alpha_{k} e_{k} w_{k}(t)$ with the $\left\{w_{k}\right\}_{k \in \mathbf{N}}$ being a family of independent two-sided standard Brownian motions defined on the probability space underlying $W$. Since $u_{N}$ is stationary, we have $\mathcal{L}\left(u_{N}(t)\right) \equiv \mathcal{Q}_{N}$ for any $t \in \mathbf{R}$. By $\mathcal{P}_{N}^{[0, T]}$ we denote the path measure of $\left\{u_{N}(t)\right\}_{t \in[0, T]}$, and by $\mathcal{P}_{N}$ the measure for the whole process $u_{N}$ in path space.

It is well-known (see e.g. [DPZ92]) that, for any pair $t>t_{0}$, the process $u_{N}$ satisfies (with probability 1 ) the following variation of constants formula:

$$
u_{N}(t)=e^{\left(t-t_{0}\right) A} u_{N}\left(t_{0}\right)+\int_{t_{0}}^{t} \partial_{x}^{2} e^{(t-s) A} \Pi_{N}\left(\partial_{x} u_{N}(s)\right)^{2} d s+\int_{t_{0}}^{t} e^{(t-s) A} d W_{N}(s) .
$$

Again, we consider the differential operators either as operators on the range of $\Pi_{N}$ or as $N \times N$-matrices.

As our solutions of (1.1) do not have enough regularity, we will focus on mild solutions, which are solutions of such integral equations. Our main result is

Theorem 2.3 Consider equation (1.1) with periodic or Neumann b.c. in the stable and only Neumann b.c. in the unstable case. Then the family of measures $\left\{\mathcal{Q}_{N}\right\}_{N \in \mathbf{N}}$ given by Proposition 2.2 is tight on $\mathrm{L}^{2}$.

Furthermore, for any of its accumulation points $\mathcal{Q}$, there exists a probability space $(\tilde{\Omega}, \tilde{\mathcal{F}}, \tilde{\mathcal{P}})$, a two-sided $Q$-Wiener process $\tilde{W}$, and a stationary stochastic process $\{u(t)\}_{t \in \mathbf{R}}$ with $u \in \mathcal{C}\left(\mathbf{R}, H^{-3}\right) \cap \mathrm{L}_{\text {loc }}^{2}\left(\mathbf{R}, H^{1}\right)$, such that $\mathcal{L}(u(t)) \equiv \mathcal{Q}$ for every $t \in \mathbf{R}$, and such that

$$
u(t)=e^{\left(t-t_{0}\right) A} u\left(t_{0}\right)+\int_{t_{0}}^{t} \partial_{x}^{2} e^{(t-s) A}\left(\partial_{x} u(s)\right)^{2} d s+\int_{t_{0}}^{t} e^{(t-s) A} d \tilde{W}(s)
$$

holds for all $t \geq t_{0}, \tilde{\mathcal{P}}$-almost surely.

We will not focus on optimal regularity, but we could slightly improve the regularity of $u$ analogous to Corollary 3.2 and 3.3 of [BG02]. Moreover, we could prove that support of the measure $\mathcal{Q}$ is concentrated in a smaller space than $\mathrm{L}^{2}$, but we are far from getting enough regularity to prove pathwise uniqueness.

In the stable case, it is easily possible to prove an analog of Theorem 2.3 with Dirichlet boundary conditions, but we do not enter into details here.

\section{Proof of the main result}

The main step of the proof of Theorem 2.3 is a bound on the logarithmic moments of $\mathcal{Q}_{N}$ that does not depend on $N$. The main technical difficulty is that Itô's formula can not be applied to (1.1) since the covariance of our noise is not necessarily trace class. We postpone the proof of Theorem 3.1 below to sections 4 and 5 . 
Theorem 3.1 Let $\mathcal{Q}_{N}$ be the measure on $\mathrm{L}^{2}$ invariant for the Nth Galerkin approximation. Then, there exists a constant $C$ independent of $N$ such that

$$
\int_{\mathrm{L}^{2}} \log \left(1+\|u\|_{\mathcal{C}^{1}}^{2}\right) \mathcal{Q}_{N}(d u) \leq C,
$$

uniformly in $N$.

Using this result, we turn to the

Proof of Theorem 2.3. The tightness of the family $\left\{\mathcal{Q}_{N}\right\}$ follows immediately from Theorem 3.1 and the compact embedding of $\mathcal{C}^{1}$ into $\mathrm{L}^{2}$. We choose any accumulation point $\mathcal{Q}$ of $\left\{\mathcal{Q}_{N}\right\}$ and assume without loss of generality that $\mathcal{Q}_{N}$ converges weakly to $\mathcal{Q}$ in the space of Borel measures on $\mathrm{L}^{2}$. Denote by $\mathcal{P}_{N}$ the law of the (unique in law) stationary process associated to the invariant measure $\mathcal{Q}_{N}$ by Proposition 2.2.

In order to construct the process $u$ appearing in the statement, we first show that the family of measures $\left\{\mathcal{P}_{N}\right\}$ is tight (it turns out that it is so on the space $\mathcal{C}\left(\mathbf{R}, H^{-3}\right) \cap$ $\mathrm{L}_{\text {loc }}^{2}\left(\mathbf{R}, H^{1}\right)$ ), and then verify that the limiting process obtained by the usual ProkhorovSkohorod argument really satisfies the integral equation (2.4).

To prove the tightness of the family $\left\{\mathcal{P}_{N}\right\}$, we consider $u_{N}$ as a solution of (2.3) with initial condition $u_{N}(0)$ distributed according to $\mathcal{Q}_{N}$. We denote by $W_{A}^{N}(t)$ the stochastic convolution given by

$$
W_{A}^{N}(t)=\int_{0}^{t} e^{A(t-s)} d W_{N}(s),
$$

and we define $v_{N}(t):=u_{N}(t)-W_{A}^{N}(t)$. The reason is that the stochastic process $v_{N}$ exhibits trajectories with much more time-regularity than $u_{N}$. The process $v_{N}$ is then pathwise a strong solution of the random PDE given by

$$
\partial_{t} v_{N}=A v_{N}-\partial_{x}^{2} \Pi_{N}\left(\partial_{x} v_{N}+\partial_{x} W_{A}^{N}\right)^{2}, \quad v_{N}(0)=u_{N}(0) .
$$

We will need the following technical lemma, the proof of which is postponed to sections 4 and 5 .

Lemma 3.2 Fix $\varepsilon, T>0$ and assume that there exists $R>0$ such that

$$
\mathbf{P}\left(\left\|u_{N}(0)\right\|>R\right)<\varepsilon \quad \text { for all } N \in \mathbf{N} .
$$

Assume furthermore that $u(0)$ is independent of the Wiener increments for positive times. Then there exists $\tilde{R}>0$ independent of $N$ such that

$$
\mathbf{P}\left(\left\|v_{N}\right\|_{\mathcal{C}\left(0, T, \mathrm{~L}^{2}\right)}+\left\|v_{N}\right\|_{\mathrm{L}^{2}\left(0, T, H^{2}\right)}>\tilde{R}\right)<\varepsilon \quad \text { for all } N \in \mathbf{N} .
$$

Using this result, we verify the tightness of $\left\{\mathcal{P}_{N}^{[0, T]}\right\}$ on the space $\mathcal{C}\left(0, T, H^{-3}\right) \cap$ $\mathrm{L}^{2}\left(0, T, H^{1}\right)$ in a similar way as in [BG02, Section 5], so we only briefly sketch the main ideas here.

Given $\varepsilon>0$, we look for a compact set $K_{\varepsilon}$ such that $\mathcal{P}_{N}^{[0, T]}\left(K_{\varepsilon}\right)$ is bounded from below by $1-\varepsilon$ for all $N$. Combining Theorem 3.1 with Lemma 3.2, there exists $R$ such that, with probability larger than $1-\varepsilon, v_{N}$ lies in a ball of radius $R$ of 
$\mathcal{C}\left(0, T, \mathrm{~L}^{2}\right) \cap \mathrm{L}^{2}\left(0, T, H^{2}\right)$ and $\left\|v_{N}(0)\right\| \leq R$. Furthermore, $W_{A}^{N} \rightarrow W_{A}$ in $\mathcal{C}\left(0, T, \mathcal{C}^{1}\right)$ $\mathcal{P}$-a.s. Using standard compactness results (e.g. [Gat93, Proposition 1] or [DPZ92, Proposition 8.4]) for the integral operator appearing in (2.3), we can check that the above bounds imply the existence of a compact subset $K_{\varepsilon}^{1}$ of $C\left(0, T, H^{-3}\right)$ such that $v_{N} \in K_{\varepsilon}^{1}$ with probability larger than $1-\varepsilon$. Since $v_{N}$ is also bounded in $\mathrm{L}^{2}\left(0, T, H^{2}\right)$ with high probability, we obtain by an interpolation theorem (e.g. [VF88, Theorem IV.4.1]) the existence of a compact set $K_{\varepsilon}^{2} \subset \mathrm{L}^{2}\left(0, T, H^{1}\right)$ such that $v_{N} \in K_{\varepsilon}^{2}$ with probability larger than $1-\varepsilon$.

Hence, $\left\{\mathcal{P}^{v_{N}}\right\}_{N \in \mathbf{N}}$ is tight on the space $\mathcal{C}\left(0, T, H^{-3}\right) \cap \mathrm{L}^{2}\left(0, T, H^{1}\right)$. By the definition of the projection $\Pi^{N}$, we readily obtain the convergence of $W_{A}^{N}=\Pi^{N} W_{A} \rightarrow W_{A}$ in $\mathcal{C}\left(0, T, H^{1}\right)$, as $W_{A}$ is already in that space. Combining both arguments, we thus obtain the tightness of the family $\left\{\mathcal{P}_{N}^{[0, T]}\right\}_{N \in \mathbf{N}}$ on the space $\mathcal{C}\left(0, T, H^{-3}\right) \cap \mathrm{L}^{2}\left(0, T, H^{1}\right)$. Since this holds for arbitrary time intervals, it is straightforward to extend this to the whole line, so $\left\{\mathcal{P}_{N}\right\}$ is tight on $\mathcal{C}\left(\mathbf{R}, H^{-3}\right) \cap \mathrm{L}_{\text {loc }}^{2}\left(\mathbf{R}, H^{1}\right)$. We call $\mathcal{P}^{*}$ one of its limiting measures and we obtain a subsequence $\left\{\mathcal{P}_{N_{k}}\right\}$ that converges weakly to $\mathcal{P}^{*}$ in the abovementioned space.

Now we can use Skohorod's Theorem to obtain a new probability space $(\tilde{\Omega}, \tilde{\mathcal{F}}, \tilde{\mathcal{P}})$, a $Q$-Wiener process $\tilde{W}$ on that space, stochastic processes $\tilde{u}_{k}$ with laws $\tilde{\mathcal{P}}_{k}=\mathcal{P}_{N_{k}}$ solving (2.3) with $\Pi_{N_{k}} \tilde{W}$ instead of $W_{N}$, as well as a stochastic process $\tilde{u}$ with probability distribution $\mathcal{L}(\tilde{u})=\mathcal{P}^{*}$ such that $\tilde{u}_{k} \rightarrow \tilde{u} \tilde{\mathcal{P}}$-a.s. in $\mathrm{L}_{\text {loc }}^{2}\left(\mathbf{R}, H^{1}\right) \cap \mathcal{C}\left(\mathbf{R}, H^{-3}\right)$. Hence, $\tilde{u}_{k}(t) \rightarrow \tilde{u}(t)$ in $H^{-3}$, and additionally we have $\tilde{\mathcal{P}}^{t}=\mathcal{Q}$ for all $t \in \mathbf{R}$ by our initial choice of a subsequence.

To show that $\tilde{u}$ is actually stationary, we first remark that $\tilde{u}_{k} \rightarrow \tilde{u}$ in $\mathcal{C}\left(\mathbf{R}, H^{-3}\right)$. Hence, for any choice of $\left(t_{1}, \ldots, t_{m}\right) \in \mathbf{R}^{m}$ we readily obtain in the weak convergence of measures on $\left(H^{-3}\right)^{m}$ that $\mathcal{L}\left(\left(\tilde{u}_{k}\left(t_{1}\right), \ldots, \tilde{u}_{k}\left(t_{m}\right)\right) \rightarrow \mathcal{L}\left(\left(\tilde{u}\left(t_{1}\right), \ldots, \tilde{u}\left(t_{m}\right)\right)\right.\right.$. Since $\tilde{u}_{k}$ is stationary, this immediately implies the stationarity of $\tilde{u}$.

Using the $\tilde{\mathcal{P}}$-a.s. convergence as in [BG02, Theorem 3.1], it is technical but straightforward to verify that $\tilde{u}$ is actually a solution of (2.4) with respect to $\tilde{W}$. This completes the proof of Theorem 2.3.

\section{The stable case}

This section provides the postponed proofs of the previous sections in the case of strictly negative $A$. We will discuss the necessary changes in order to cover the unstable case in Section 5 below. We start with the

Proof of Proposition 2.2. The claim follows from [DPZ96, Has80, if we can show that there exists a constant $C$ such that $\mathbf{E}\left\|u_{N}(t)\right\|^{2} \leq C$ uniformly in $t$. By Itô's formula, we have

$$
d\left\|u_{N}\right\|^{2}=2\left\langle u_{N}, A u_{N}\right\rangle d t+2\left\langle u_{N}, \partial_{x}^{2}\left(\partial_{x} u_{N}\right)^{2}\right\rangle d t+2\left\langle u_{N}, d W_{N}(t)\right\rangle+\sum_{k=1}^{N} \alpha_{k}^{2} d t
$$

Since $\left\langle u_{N}, \partial_{x}^{2}\left(\partial_{x} u_{N}\right)^{2}\right\rangle=0$ and $A$ is a strictly negative definite operator, the claim follows after integrating 4.1) on both sides, taking expectations, and applying Gronwall's formula. Notice that the bound on the second momenta obtained with this procedure 
diverges with $N$ and it remains an open problem to establish a bound independent of $N$ for arbitrary solutions.

To prove Theorem 3.1 for the stable case, we first verify an $\mathrm{L}^{2}$-bound.

Theorem 4.1 Let $\mathcal{Q}_{N}$ be the invariant measure on $\mathrm{L}^{2}$ for the Nth Galerkin approximation. There exists a constant $C$ such that

$$
\int_{\mathrm{L}^{2}} \log \left(1+\|u\|^{2}\right) \mathcal{Q}_{N}(d u) \leq C,
$$

for all $N$.

Proof. By (3.1), the $\mathrm{L}^{2}$-norm $\left\|v_{N}(t)\right\|^{2}$ satisfies

$$
\begin{aligned}
\partial_{t}\left\|v_{N}\right\|^{2}= & 2\left\langle v_{N}, A v_{N}\right\rangle-2\left\langle v_{N}, \partial_{x}^{2}\left(\partial_{x} v_{N}+\partial_{x} W_{A}^{N}\right)^{2}\right\rangle \\
= & 2\left\langle v_{N}, A v_{N}\right\rangle-2 \int_{0}^{L} \partial_{x}\left(\partial_{x} v_{N}\right)^{2} d x-4 \int_{0}^{L} \partial_{x}^{2} v_{N} \partial_{x} v_{N} \partial_{x} W_{A}^{N} d x \\
& \quad-2 \int_{0}^{L} \partial_{x}^{2} v_{N}\left(\partial_{x} W_{A}^{N}\right)^{2} d x \\
\leq & -\left\|\partial_{x}^{2} v_{N}\right\|^{2}-\nu\left\|\partial_{x} v_{N}\right\|^{2}+4\left\|\partial_{x}^{2} v_{N}\right\|\left\|\partial_{x} v_{N}\right\|\left\|\partial_{x} W_{A}^{N}\right\|_{\infty} \\
& \quad+2\left\|\partial_{x}^{2} v_{N}\right\|\left\|\partial_{x} W_{A}^{N}\right\|_{4}^{2} \\
\leq & -\frac{1}{4}\left\|\partial_{x}^{2} v_{N}\right\|^{2}-\nu\left\|\partial_{x} v_{N}\right\|^{2}+8\left\|v_{N}\right\|^{2}\left\|\partial_{x} W_{A}^{N}\right\|_{\infty}^{4}+4\left\|\partial_{x} W_{A}^{N}\right\|_{4}^{4}
\end{aligned}
$$

Using the Poincare inequality and the fact that we consider only solutions with vanishing mean, we see that there exists a positive constant $\alpha$ independent of $N$ (but depending on $L$ ) such that

$$
\partial_{t}\left\|v_{N}\right\|^{2} \leq-\alpha\left\|v_{N}\right\|^{2}+8\left\|v_{N}\right\|^{2}\left\|\partial_{x} W_{A}^{N}\right\|_{\infty}^{4}+4\left\|\partial_{x} W_{A}^{N}\right\|_{4}^{4}
$$

We define now for any interval $[s, t]$ the quantity $W_{[s, t]}^{N}$ by

$$
W_{[s, t]}^{N}=\int_{s}^{t} 8\left\|\partial_{x} W_{A}^{N}(r)\right\|_{\infty}^{4} d r .
$$

As a consequence, we have the following a-priori estimate on the norm of $v_{N}$ :

$$
\begin{aligned}
\left\|v_{N}(t)\right\|^{2} & \leq e^{-\alpha t+W_{[0, t]}^{N}}\left\|v_{N}(0)\right\|^{2}+C \int_{0}^{t} e^{-\alpha(t-s)+W_{[s, t]}^{N}}\left(1+\left\|\partial_{x} W_{A}^{N}(s)\right\|_{4}^{4}\right) d s \\
& \leq e^{-\alpha t+W_{[0, t]}^{N}}\left\|v_{N}(0)\right\|^{2}+C e^{W_{[0, t]}^{N}}\left(W_{[0, t]}^{N}+t\right) .
\end{aligned}
$$

Since $u_{N}=v_{N}+W_{A}^{N}$ and $W_{A}^{N}(0)=0$ we obtain for some $\varepsilon>0$ fixed later on:

$$
\begin{aligned}
\left\|u_{N}(t)\right\|^{2} & \leq(1+\varepsilon)\left\|v_{N}(t)\right\|^{2}+C\left\|W_{A}^{N}(t)\right\|^{2} \\
& \leq(1+\varepsilon) e^{-\alpha t+W_{[0, t]}^{N}}\left\|u_{N}(0)\right\|^{2}+C e^{W_{[0, t]}^{N}}\left(W_{[0, t]}^{N}+t\right)+C\left\|W_{A}^{N}(t)\right\|^{2} .
\end{aligned}
$$


Note that the constants may depend on $\varepsilon$.

The problem at this point is that the exponential moment of the random variable $W_{[0, t]}^{N}$ is infinite. We therefore take logarithms on both sides, yielding

$$
\begin{aligned}
\log \left(1+\left\|u_{N}(t)\right\|^{2}\right) \leq \log ((1 & +\varepsilon) e^{-\alpha t+W_{[0, t]}^{N}\left(1+\left\|u_{N}(0)\right\|^{2}\right)} \\
& \left.+C e^{W_{[0, t]}^{N}}\left(W_{[0, t]}^{N}+t\right)+C\left\|W_{A}^{N}(t)\right\|^{2}+1\right) .
\end{aligned}
$$

Using Lemma 6.1 it is now easy to verify that we can apply Lemma 6.4 (with a constant $K$ independent of $N$ ) to the right-hand side of (4.4), where we take the conditional expectation w.r.t. $u_{N}(0)$. Hence,

$$
\begin{aligned}
& \mathbf{E}\left(\left(\log \left(1+\left\|u_{N}(t)\right\|^{2}\right)\right)^{2} \mid u_{N}(0)\right) \leq\left(\log \left(1+\left\|u_{N}(0)\right\|^{2}\right)\right)^{2} \\
& \quad+2\left(\varepsilon+\log (1+\varepsilon)-\alpha t+\mathbf{E} W_{[0, t]}^{N}\right) \cdot \log \left(1+\left\|u^{N}(0)\right\|^{2}\right)+C
\end{aligned}
$$

for some constant $C$ depending on $\varepsilon$ and on the parameters of the problem, but not on $N$.

At this point, we choose first $t$ sufficiently small such that

$$
\mathbf{E} W_{[0, t]}^{N} \leq \frac{\alpha t}{2}
$$

This can be done uniformly in $N$ by Lemma 6.1. Then fix $\varepsilon$ so small such that

$$
\varepsilon+\log (1+\varepsilon) \leq \frac{\alpha t}{4} .
$$

Taking expectations on both sides of (4.5) and using the stationarity of $u_{N}(t)$, we have

$$
\mathbf{E} \log \left(1+\left\|u_{N}(0)\right\|^{2}\right) \leq \frac{C}{\alpha t}
$$

for fixed $t$ sufficiently small, therefore concluding the proof of Theorem 4.1 .

Remark 4.2 Theorem 4.1 establishes only a uniform bound (in $t$ ) for stationary solutions of our problem. Estimates like (4.4) or (4.5) are not sufficient to get uniform bounds for arbitrary solutions, and this question remains open.

Let us now turn to the

Proof of Theorem 3.1. Using (4.2) we obtain after integration

$$
\begin{aligned}
\int_{0}^{1}\left\|\partial_{x}^{2} v_{N}(t)\right\|^{2} d t \leq & C \int_{0}^{1}\left\|v_{N}(t)\right\|^{2}\left(1+\left\|\partial_{x} W_{A}^{N}(t)\right\|_{\infty}^{4}\right) d t \\
& +C \int_{0}^{1}\left\|\partial_{x} W_{A}^{N}(t)\right\|_{4}^{4} d t+C\left\|v_{N}(0)\right\|^{2}
\end{aligned}
$$


Using Young's inequality and the Sobolev embedding of $H^{1}$ into $\mathrm{L}^{\infty}$, we have the bound

$$
\left\|u_{N}\right\|_{\mathcal{C}^{1}}^{2} \leq C\left\|\partial_{x}^{2} v_{N}\right\|^{2}+C\left\|\partial_{x} W_{A}^{N}\right\|_{\infty}^{4}+C
$$

This yields

$$
\begin{aligned}
& \int_{0}^{1}\left\|u_{N}(t)\right\|_{\mathcal{C}^{1}}^{2} d t \leq C \int_{0}^{1}\left\|v_{N}(t)\right\|^{2}\left(1+\left\|\partial_{x} W_{A}^{N}(t)\right\|_{\infty}^{4}\right) d t \\
& +C \int_{0}^{1}\left\|\partial_{x} W_{A}^{N}(t)\right\|_{4}^{4} d t+C+C\left\|u_{N}(0)\right\|^{2} \\
& \leq C \int_{0}^{1}\left(e^{-\alpha t+W_{[0, t]}^{N}}\left\|u_{N}(0)\right\|^{2}+e^{W_{[0, t]}^{N}}\left(W_{[0, t]}^{N}+t\right)\right) \\
& \times\left(1+\left\|\partial_{x} W_{A}^{N}(t)\right\|_{\infty}^{4}\right) d t+C \int_{0}^{1}\left\|\partial_{x} W_{A}^{N}(t)\right\|_{4}^{4} d t+C+C\left\|u_{N}(0)\right\|^{2} \\
& \leq C\left(\left(e^{W_{[0,1]}^{N}}+1\right)\left\|u_{N}(0)\right\|^{2}+e^{W_{[0,1]}^{N}}\left(W_{[0,1]}^{N}+1\right)+1\right)\left(1+W_{[0,1]}^{N}\right)
\end{aligned}
$$

where we used (4.3). Using Theorem 4.1 and Lemma 6.1, we immediately obtain

$$
\mathbf{E} \log \left(\int_{0}^{1}\left\|u_{N}(t)\right\|_{\mathcal{C}^{1}}^{2} d t+1\right) \leq C .
$$

Finally, Jensen's inequality and the stationarity of $u_{N}$ yield

$$
\begin{aligned}
\mathbf{E} \log \left(\left\|u_{N}(0)\right\|_{\mathcal{C}^{1}}^{2}+1\right) & =\mathbf{E} \int_{0}^{1} \log \left(\left\|u_{N}(t)\right\|_{\mathcal{C}^{1}}^{2}+1\right) d t \\
& \leq \mathbf{E} \log \left(\int_{0}^{1}\left\|u_{N}(t)\right\|_{\mathcal{C}^{1}}^{2} d t+1\right) \leq C,
\end{aligned}
$$

concluding the proof of Theorem 3.1 .

We now turn to the proof of Lemma 3.2. This proof will not use the strict negativity of $A$ and is thus still valid in the unstable case. Since we need this bound only for a fixed time interval $[0, T]$, we can bound the terms in a rather crude way.

Proof of Lemma 3.2. Define

$$
W_{T}^{N}:=\sup _{t \in[0, T]}\left\|\partial_{x} W_{A}^{N}(t)\right\|_{\infty}^{4}
$$

Using the factorization method and Sobolev embedding it is straightforward to check that $\mathbf{E} W_{T}^{N}<C$ uniformly in $N$. This result is established completely analogous to [BMPS01, Lemma 5.1]. Note that the uniformity in $N$ is not trivial, as the family $\left\{\Pi^{N}\right\}_{N \in \mathbf{N}}$ is not uniformly bounded as operators on $L^{\infty}$ or $\mathcal{C}^{0}$.

Using this and the assumption on $\left\|u_{N}(0)\right\|$, we see that for every $\varepsilon>0$ there is an $R>0$ such that

$$
\mathbf{P}\left(W_{T}^{N}<R \quad \text { and } \quad\left\|u_{N}(0)\right\|<R\right)>1-\varepsilon
$$


Combining (4.7) and (4.3), we see that with probability larger than $1-\varepsilon$ one has

$$
\begin{aligned}
\left\|v_{N}(t)\right\|^{2} & \leq e^{W_{T}^{N}}\left\|v_{N}(0)\right\|^{2}+C e^{W_{T}^{N}}\left(W_{T}^{N}+T\right) \\
& \leq e^{R} R^{2}+C e^{R}(R+T)=: R^{\prime},
\end{aligned}
$$

for any $t \in[0, T]$. Using (4.6) in the same way, we get

$$
\begin{aligned}
\int_{0}^{T}\left\|\partial_{x}^{2} v_{N}(t)\right\|^{2} d t & \leq C\left\|v_{N}(0)\right\|^{2}+C \int_{0}^{T}\left\|v_{N}(t)\right\|^{2}\left(W_{T}^{N}+1\right) d t+C T W_{T}^{N} \\
& \leq C\left(R+T R^{\prime}(R+1)+T R\right),
\end{aligned}
$$

with probability larger than $1-\varepsilon$, thus concluding the proof of Lemma 3.2.

\section{The unstable case}

This section deals with the case where the operator $A$ is no longer strictly negative definite. In order to treat this case, we make use of a trick that was used in [NST85, CEES93, to get bounds on the deterministic Kuramoto-Sivashinsky equation. It turns out that the present model is sufficiently close to that equation to make that trick go through. Nevertheless, we can only treat Neumann boundary conditions (which is the same as considering the restriction on $[0, L]$ of functions that are even and periodic with period $2 L$ ). In a similar way we can treat also Dirichlet boundary conditions, but periodic boundary conditions are still open.

Most of the proofs are similar to the previous section, so we will only state the main differences. Instead of defining $v_{N}$ as previously, we define $v_{N}$ by

$$
v_{N}(t)=u_{N}(t)-W_{A}^{N}(t)-\Phi_{N},
$$

where $\Phi_{N}=\Pi_{N} \Phi$ for some function $\Phi$ to be chosen later and $W_{A}^{N}$ is the stochastic convolution defined in the previous section. The stochastic process $v_{N}$ then satisfies the following random PDE:

$$
\partial_{t} v_{N}=A v_{N}+A \Phi_{N}-\Pi_{N} \partial_{x}^{2}\left(\partial_{x} v_{N}+\partial_{x} W_{A}^{N}+\partial_{x} \Pi_{N} \Phi\right)^{2} .
$$

We can rewrite this as

$$
\partial_{t} v_{N}=\tilde{A} v_{N}-\partial_{x}^{2}\left(\partial_{x} v_{N}+\partial_{x} W_{A}^{N}\right)^{2}-\partial_{x}^{2}\left(\partial_{x} \Pi_{N} \Phi\right)^{2}+A \Phi-2 \partial_{x}^{2}\left(\partial_{x} \Phi \partial_{x} W_{A}\right),
$$

where the operator $\tilde{A}$ is defined as

$$
\tilde{A} v=A v-2 \partial_{x}^{2}\left(\partial_{x} \Phi \partial_{x} v\right) .
$$

Using exactly the same technique as in the previous section, we see that in order to get uniform bounds on the Galerkin approximations of $v$, it suffices to find a smooth function $\Phi$ such that

$$
\langle v, \tilde{A} v\rangle \leq-c\left\|\partial_{x}^{2} v\right\|^{2}
$$

for some constant $c>0$. Using this function, it is easy to verify that the assertions of Proposition 2.2 and Theorems 3.1 and 4.1 hold in the unstable case, too. The only major 
changes appear in the values of the constants, which do now depend on the choice of $\Phi$. We will therefore not go through the proofs of these assertions for the unstable case, but we will sketch how to find a function $\Phi$ such that $\tilde{A}$ is strictly negative definite.

Integrating by parts, we see that the bilinear form (5.3) can be written as

$$
\langle v, \tilde{A} v\rangle=-\left\|\partial_{x}^{2} v\right\|^{2}-\nu\left\|\partial_{x} v\right\|^{2}-\left\langle\partial_{x} v,\left(\partial_{x}^{2} \Phi\right) \partial_{x} v\right\rangle,
$$

where $\nu$ is negative. The problem is therefore reduced to finding a smooth periodic function $\Phi$ such that the Schrödinger operator

$$
\mathcal{H}_{\Phi}=-\frac{1}{2} \partial_{x}^{2}+\partial_{x}^{2} \Phi(x),
$$

with Dirichlet boundary conditions satisfies $\left\langle u, \mathcal{H}_{\Phi} u\right\rangle \geq|\nu|\|u\|^{2}$ for all functions $u$ in its domain. The idea appearing in [NST85] is to choose $\Phi$ such that, away from the boundary, $\partial_{x}^{2} \Phi$ is for all practical purposes constant and sufficiently large (say equal to about $2|\nu|)$. The problem is that, in order for (5.2) to hold, $\Phi$ has to belong to $D(A)$ and must therefore satisfy the same boundary conditions as $u$. As a consequence $\partial_{x}^{2} \Phi$ must satisfy $\int_{0}^{L} \partial_{x}^{2} \Phi(s) d x=0$, which is of course impossible for a constant (non-zero) function. Looking at (5.4), we notice that $\partial_{x}^{2} \Phi(x)=2|\nu|(1-\delta(x))$ would formally fit our needs, since the delta-peak is integrated against $\partial_{x} v$, which vanishes at the boundaries, due to the Neumann conditions. The function $\Phi$ obtained this way does of course not belong to $D(A)$, so we look for an approximation of it which is more regular.

Since $\Phi$ satisfies Neumann boundary conditions, it is natural to write it as

$$
\Phi(x)=2|\nu| \sum_{n=1}^{\infty} \varphi_{n} \cos \left(\frac{2 \pi n}{L}\right) .
$$

(The sum starts at 1 because we are interested only in functions with vanishing mean.) If we choose $\varphi_{n}=2 n^{-2}$, we see that $\partial_{x}^{2} \Phi$ is given by $\partial_{x}^{2} \Phi(x)=2|\nu|(1-\delta(x))$, which is what we would like to approximate. In order to get a regular function, we define

$$
\psi_{n}:=n^{2} \varphi_{n}= \begin{cases}2 & \text { for } n \leq 2 n_{*}, \\ 0 & \text { for } n>2 n_{*},\end{cases}
$$

where $n_{*}$ is some (sufficiently large) constant to be fixed later on. With this definition, we have:

Proposition 5.1 For every $L, C>0$, there exists a value $n_{*}>0$ such that the quadratic form $\mathcal{H}_{\Phi}$ with $\Phi$ defined as in (5.5) and (5.6), satisfies

$$
\int_{0}^{L} u(x)\left(\mathcal{H}_{\Phi} u\right)(x) d x \geq C\|u\|^{2},
$$

for every $u$ in the domain of $\mathcal{H}_{\Phi}$.

Remark 5.2 Notice that the function $\Phi$ defined by $(5.5)$ and $(5.6)$ is actually analytic, so the expressions appearing in (5.2) and containing $\Phi$ can all be bounded uniformly in $N$ (not in $n_{*}$ of course, but $n_{*}$ is chosen independently of $N$ ). 
Remark 5.3 As in [CEES93] we could choose a slow decay of $\psi_{n}$ for $n>2 n_{*}$ to optimize the $L$-dependence of our bound, but we neglected this for simplicity.

Proof. Applying the arguments of [CEES93, Prop. 2.1], we see that it suffices to show that the quantity

$$
\Gamma:=\sum_{k>m>0} \frac{\left|\psi_{k+m}-\psi_{k-m}\right|^{2}}{E_{k} E_{m}}, \quad \text { with } \quad E_{n}=\alpha n^{2}, \quad \alpha=\frac{2 \pi^{2}}{L^{2}},
$$

can be made arbitrarily small by choosing $n_{*}$ sufficiently large. The only non-vanishing terms of this sum are those where $0 \leq k-m \leq 2 n_{*}$ and $k+m \geq 2 n_{*}$. We can estimate these terms by

$$
\begin{aligned}
\Gamma & \leq \frac{4}{\alpha^{2}} \sum_{m=1}^{n_{*}-1} \sum_{k=2 n_{*}-m}^{2 n_{*}+m} \frac{1}{m^{2} k^{2}}+\frac{4}{\alpha^{2}} \sum_{m=n_{*}}^{\infty} \sum_{k=m}^{2 n_{*}+m} \frac{1}{m^{2} k^{2}} \\
& \leq \frac{8}{\alpha^{2} n_{*}} \sum_{m=1}^{n_{*}-1} \frac{1}{m^{2}}+\frac{8}{\alpha^{2} n_{*}} \sum_{m=n_{*}}^{\infty} \frac{1}{m^{2}} \leq \frac{4 \pi^{2}}{3 \alpha^{2} n_{*}} .
\end{aligned}
$$

In both sums, we used the fact that $k$ is larger than $n_{*}$ and that there are less than $2 n_{*}$ terms in the inner sum. Thus, $\Gamma$ can clearly be made arbitrarily small by choosing $n_{*}$ sufficiently large. This proves Proposition 5.1 and concludes our exposition of the unstable case.

\section{Technical estimates}

In this section, we prove the two technical estimates required for the proof of Theorem 4.1 above.

Lemma 6.1 There exists a constant $C$ independent of $N$ such that

$$
\mathbf{E}\left\|\partial_{x} W_{A}^{N}(t)\right\|_{\infty}^{4} \leq C t^{1 / 8}
$$

for all $t \leq 1$.

Remark 6.2 The power $1 / 8$ in the above lemma is not optimal but it is sufficient for our needs. All we need is $\mathbf{E}\left\|\partial_{x} W_{A}^{N}(t)\right\|_{\infty}^{4}=o(1)$ uniformly in $N$.

Remark 6.3 The constant in the above lemma depends only on the coefficients of the problem and the bound on the $\alpha_{k}$. It is possible to allow for slowly growing $\alpha_{k}$, using the Sobolev embedding of $\mathrm{L}^{\infty}$ into the fractional Sobolev space $W^{s, p}$ for $s p>1$.

Proof. For $f \in \mathrm{L}^{\infty}([0, L])$ with vanishing mean, denote by $\left\{f_{k}\right\}_{k \in \mathbf{N}}$ its Fourier coefficients. Since the eigenfunctions $e_{k}$ of $A$ are uniformly bounded in $\mathrm{L}^{\infty}$, we have the following estimate on $\|f\|_{\infty}$ :

$$
\|f\|_{\infty} \leq C \sum_{k=1}^{\infty}\left|f_{k}\right| \leq C\left(\sum_{k=1}^{\infty}|k|^{1 / 2}\left|f_{k}\right|^{4 / 3}\right)^{3 / 4}\left(\sum_{k=1}^{\infty}|k|^{-3 / 2}\right)^{1 / 4} \leq C\|K f\|_{4},
$$


where $K$ is the operator that acts on Fourier coefficients as $(K f)_{k}=|k|^{3 / 8} f_{k}$. Here we used the usual isometry between $\mathrm{L}^{p}$ and $\ell^{q}$ for $p^{-1}+q^{-1}=1$.

Denote by $\lambda_{k}$ the eigenvalues corresponding to the eigenfunctions $e_{k}$. By the definition of $A$, there exist constants $c_{i}$ such that

$$
c_{1} k^{4} \leq-\lambda_{k} \leq c_{2} k^{4}, \quad\left|\left(K \partial_{x} e_{k}\right)(x)\right|^{2} \leq c_{3} k^{11 / 4} .
$$

With these notations, $\left(K \partial_{x} W_{A}^{N}\right)(t, x)$ (with fixed values of $x$ and $t$ ) are centered Gaussian random variables given by

$$
\left(K \partial_{x} W_{A}^{N}\right)(t, x)=\sum_{k=1}^{N} \alpha_{k}\left(K \partial_{x} e_{k}\right)(x) \int_{0}^{t} e^{-\lambda_{k}(t-s)} d w_{k}(s),
$$

with independent Wiener processes $w_{k}(t)$. The variance of $\left(K \partial_{x} W_{A}^{N}\right)(t, x)$ is thus bounded by

$$
\begin{aligned}
\mathbf{E}\left|\left(K \partial_{x} W_{A}^{N}\right)(t, x)\right|^{2} & =\sum_{k=1}^{N} \alpha_{k}^{2}\left|\left(K \partial_{x} e_{k}\right)(x)\right|^{2} \int_{0}^{t} e^{2 \lambda_{k}(t-s)} d s \\
& \leq C \cdot \sum_{k=1}^{\infty}|k|^{11 / 4} \int_{0}^{t} e^{-c_{1} k^{4} s} d s \leq \sum_{k=1}^{\infty} \frac{C}{k^{5 / 4}}\left(1-e^{-c_{2} k^{4} t}\right) .
\end{aligned}
$$

We now take some $k_{*}$ to fixed later and split the sum into two parts:

$$
\mathbf{E}\left|\left(K \partial_{x} W_{A}\right)(x, t)\right|^{2} \leq C \sum_{k=1}^{k_{*}-1} k^{11 / 4} t+C \sum_{k=k_{*}}^{\infty} \frac{1}{k^{5 / 4}} \leq C k_{*}^{15 / 4} t+\frac{C}{k_{*}^{1 / 4}} .
$$

Choosing $k_{*} \approx t^{-1 / 4}$, we have the estimate $\mathbf{E}\left|\left(K \partial_{x} W_{A}^{N}\right)(x, t)\right|^{2} \leq C t^{1 / 16}$. Since the random variables $\left(K \partial_{x} W_{A}^{N}\right)(x, t)$ are Gaussian for all fixed values $(x, t)$, we have

$$
\mathbf{E}\left\|\partial_{x} W_{A}^{N}\right\|_{\infty}^{4} \leq C \mathbf{E}\left\|K \partial_{x} W_{A}^{N}\right\|_{4}^{4}=\int_{0}^{L} \mathbf{E}\left(K \partial_{x} W_{A}\right)(x, t)^{4} d x \leq C t^{1 / 8}
$$

Lemma 6.4 For every $\varepsilon>0$ and $K>0$ there exists a constant $C$ depending only on $\varepsilon$ and $K$, such that for any two random variables $W_{1}$ and $W_{2}$ with $\mathbf{E}\left(\left|W_{1}\right|^{2}+\left|W_{2}\right|^{2}\right) \leq$ $K$ we obtain

$$
\mathbf{E}\left(\log \left(x e^{W_{1}}+e^{W_{2}}\right)\right)^{2} \leq(\log (x))^{2}+2\left(\varepsilon+\mathbf{E} W_{1}\right) \log x+C,
$$

for every $x \geq 1$.

Proof. Expanding the square, we get

$$
\mathbf{E}\left(\log \left(x e^{W_{1}}+e^{W_{2}}\right)\right)^{2}=(\log x)^{2}+2 \log x\left(\mathbf{E} W_{1}+\mathbf{E} \log \left(1+e^{W_{2}-W_{1}} / x\right)\right)
$$




$$
+\mathbf{E}\left(W_{1}+\log \left(1+e^{W_{2}-W_{1}} / x\right)\right)^{2}
$$

Since we assumed $x \geq 1$, we have

$$
\begin{aligned}
\mathbf{E}\left(W_{1}+\log \left(1+e^{W_{2}-W_{1}} / x\right)\right)^{2} & \leq 2\left(\mathbf{E} W_{1}^{2}+\mathbf{E}\left(W_{2}-W_{1}\right)^{2}+1\right) \\
& \leq 6 K+2
\end{aligned}
$$

It thus suffices to show that there exists $x_{0}>0$ depending only on $K$ and on $\varepsilon$ such that

$$
E_{x}:=\mathbf{E} \log \left(1+\frac{e^{W_{2}-W_{1}}}{x}\right) \leq \varepsilon
$$

for $x$ larger than $x_{0}$.

To verify (6.1) consider arbitrary $\varepsilon>0$. We define $y=\left|W_{2}-W_{1}\right|$ and denote the probability distribution on $\mathbf{R}_{+}$of $y$ by $\mathbf{P}$. Now choose $y_{0}>1$ large enough such that

$$
\int_{y_{0}}^{\infty} y \mathbf{P}(d y) \leq \varepsilon, \quad \int_{y_{0}}^{\infty} \mathbf{P}(d y) \leq \varepsilon .
$$

We choose $y_{0}=1+\left(\mathbf{E} y^{2}\right) / \varepsilon$. Now define

$$
x_{0}=\frac{e^{1+2 K / \varepsilon}}{\varepsilon} \geq \frac{e^{y_{0}}}{\varepsilon} .
$$

We thus have

$$
\begin{aligned}
E_{x} & =\int_{0}^{y_{0}} \log \left(1+\frac{e^{y}}{x}\right) \mathbf{P}(d y)+\int_{y_{0}}^{\infty} \log \left(1+\frac{e^{y}}{x}\right) \mathbf{P}(d y) \\
& \leq \log \left(1+\frac{e^{y_{0}}}{x}\right)+\int_{y_{0}}^{\infty} \log \left(1+\varepsilon e^{y-y_{0}}\right) \mathbf{P}(d y) \\
& \leq \varepsilon+\int_{y_{0}}^{\infty} \log \left((1+\varepsilon) e^{y-y_{0}}\right) \mathbf{P}(d y) \\
& \leq \varepsilon+\varepsilon \log (1+\varepsilon)+\int_{y_{0}}^{\infty}\left(y-y_{0}\right) \mathbf{P}(d y) \leq 2 \varepsilon+\varepsilon \log (1+\varepsilon) .
\end{aligned}
$$

The claim follows immediately.

\section{References}

[Arn74] L. Arnold, Stochastic Differential Equations: Theory and Applications, WileyInterscience Publication, John Wiley \& Sons, New York, 1974.

[BG02] D. Blömker and C. Gugg, On the Existence of Solutions for Amorphous Molecular Beam Epitaxy, Journal of Nonlinear Analysis: Series B Real World Applications 3 (2002), no. 1, 61-73.

[BGR02] D. Blömker, C. Gugg, and M. Raible, Thin-Film-Growth Models: Roughness and Correlation Functions, To appear in European Journal of Appl. Math., 2002. 
[BMPS01] D. Blömker, S. Maier-Paape, and G. Schneider, The Stochastic Landau Equation as an Amplitude Equation, Discrete and Continuous Dynamical Systems, Series B 1 (2001), no. 4, 527-541.

[BS95] A. L. Barabasi and H. E. Stanley, Fractal Concepts in Surface Growth, Cambridge University Press, 1995.

[CEES93] P. Collet, J.-P. Eckmann, H. Epstein, and J. Stubbe, A Global Attracting Set for the Kuramoto-Sivashinsky Equation, Commun. Math. Phys. 152 (1993), no. 1, 203214.

[CG94] M. Capinski and D. Gatarek, Stochastic Equations in Hilbert Space with Applications to Navier-Stokes Equation in any Dimension, Journal of Functional Analysis 126 (1994), no. 1, 26-35.

[DPZ92] G. Da Prato and J. Zabczyk, Stochastic Equations in Infinite Dimensions, University Press, Cambridge, 1992.

[DPZ96] G. Da Prato and J. Zabczyk, Ergodicity for Infinite Dimensional Systems, London Mathematical Society Lecture Note Series, vol. 229, University Press, Cambridge, 1996.

[FG95] F. Flandoli and D. Gatarek, Martingale and Stationary Solutions for Stochastic Navier-Stokes Solutions, Probab. Theory Relat. Fields 102 (1995), 367-391.

[Gat93] D. Gatarek, A Note on Stochastic Equations in Hilbert Spaces, Statistics and Probability Letters 17 (1993), no. 5, 387-394.

[Has80] R. Z. Has'minskiǐ, Stochastic Stability of Differential Equations, Sijthoff \& Noordhoff, 1980.

[MMS99] S. G. Mayr, M. Moske, and K. Samwer, Identification of Key Parameters by Comparing Experimental and Simulated Growth of Vapor Deposited Amorphous $\mathrm{Zr}_{65} \mathrm{Al}_{7.5} \mathrm{Cu}_{27.5}$ Films, Phys. Rev. B 60 (1999), 16950-16955.

[NST85] B. Nicolaenko, B. Scheurer, and R. Temam, Some Global Dynamical Properties of the Kuramoto-Sivashinsky Equations: Nonlinear Stability and Attractors, Phys. D 16 (1985), no. 2, 155-183.

$\left[\mathrm{RML}^{+} 00\right]$ M. Raible, S. G. Mayr, S. J. Linz, M. Moske, P. Hänggi, and K. Samwer, Amorphous Thin Film Growth: Theory Compared with Experiment, Europhysics Letters 50 (2000), 61-67.

[SP94] M. Siegert and M. Plischke, Solid-on-Solid Models of Molecular-Beam Epitaxy, Phys. Rev. E 50 (1994), 917-931.

[VF88] M. Visik and A. Fursikov, Mathematical Problems of Statistical Hydrodynamic, Kluwer, 1988. 